\title{
Health Monitoring Studies on Composite Structures for Aerospace Applications
}

\section{SAND96-0213C

\author{
George James, Dennis Roach, Bruce Hansche, Raul Meza, Nikki Robinson \\ Sandia National Laboratories \\ Albuquerque, NM 87185-0557 \\ Abstract
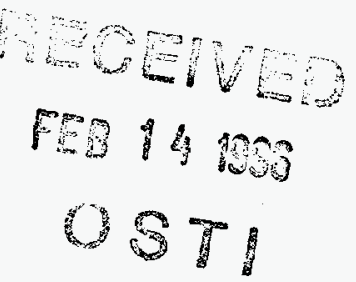

\begin{abstract}
This paper discusses ongoing work to develop structural health monitoring techniques for composite aerospace structures such as aircraft control surfaces, fuselage sections or repairs, and reusable launch vehicle fuel tanks. The overall project is divided into four tasks: operational evaluation, diagnostic measurements, information condensation, and damage detection. Five composite plates were constructed to study delaminations, disbonds, and fluid retention issues as the initial step in creating an operational system. These four square feet plates were graphite-epoxy with nomex honeycomb cores. The diagnostic measurements are composed of modal tests with a scanning laser vibrometer at over 500 scan points per plate covering the frequency range up to $2000 \mathrm{~Hz}$. This data has been reduced into experimental dynamics matrices using a generic software package developed at the University of Colorado at Boulder. The continuing effort will entail performing a series of damage identification studies to detect, localize, and determine the extent of the damage. This work is providing understanding and algorithm development for a global NDE technique for composite aerospace structures.
\end{abstract}

\section{Introduction}

Composite materials are used in a variety of aerospace applications including aircraft control surfaces, fuselage sections and repairs, and reusable launch vehicle fuel tanks. Composite structures offer numerous advantages over metallic structures including light weight, high strength, corrosion resistance, elimination of rivets, and time savings in installation. While composite structures are used extensively in military applications, their use in commercial aviation has encountered design difficulties associated with application, subsequent inspection, and long-term endurance. Also, it has been generally accepted that composite fuel tanks will be a critical element in the development of reusable launch vehicles, however rapid and reliable field-inspection techniques will be required to verify the flight status of these structures. This determination of the current state of health and/or assurance of installation requires that flaws such as disbonds, interply delaminations, fluid ingress, and adhesive failure must be located and evaluated. Because of the increasing use of composites on commercial aircraft and the potential economic impact of reusable launch systems, it appears that the demand for composite health monitoring techniques will increase.

Most composite inspections are performed with the human eye or using the non-scientific tap test. Inconsistencies in these inspection results have prompted industry to look at more advanced NonDestructive Inspection (NDI) techniques. Also, the desire to revolutionize the efficiency of these inspections has driven the recent work to develop wide area or global inspection techniques which can rapidly monitor large structures in the field. Structural dynamics provides a well understood and global set of properties to utilize in such a development. The field of Structural Health Monitoring utilizes structural dynamics properties to inspect, monitor, and assess operational structures for continued service. Development work is being performed in four areas: operational evaluation, diagnostic measurements, information condensation, and damage identification. The current status of this work and its specific application to a set of composite test articles will be the subject of this paper.

\section{Operational Evaluation}

Operational Evaluation is the process of evaluating the expected damage types, determining realistic accumulation models, and developing the appropriate test procedures for the operational structure and its environment throughout the service life. Engineered-flaw specimens, resonant fatigue testing, and ambient excitation testing have been the major developments for this aspect of the work. Only engineered-flaw specimens have been used to date in the work reported herein. Final application of structural health monitoring to structures in the will require damage accumulation studies from fatigue tests and operational evaluation tools such as ambient exciation testing. 
For this work, a series of five plates have been designed and built with a series of flaws engineered into the construction. The effects of these flaws can then be studied by comparing the response of different plates. The plates are 24 inches by 24 inches constructed of a .5 inch thick Nomex honeycomb core sandwiched between four ply T300 plain weave graphite cloth panels. The graphite lay-up is $[-45,0,90,45]$. A layer of hysol film adhesive bonds the graphite panels to the honeycomb core. Plate \#1 has no engineered flaw and is considered the undamaged specimen. Plate \#2 has a four inch diameter disbond (created with a teflon disk) in the geometric center of one graphite panel. Plate \#3 has a four inch diameter region of the honeycomb core (located in the geometric center of the plate) filled with fluid. The individual honeycomb cells surrounding the fluid are potted to contain it. Plate \#4 uses a teflon insert to produce a four inch diameter delamination between plies 2 and 3 at the geometric center of one graphite panel. Plate \#5 contains two of the four inch diameter disbonds located at the geometric centers of opposing quadrants of a graphite panel. A four inch diameter delamination, and a four inch diameter fluid ingress section are at the geometric centers of the two remaining quadrants. Figure 1 shows a schematic for plate \#2. Initial results from this plate will be shown in a later section. These three types of flaws in the plates represent common flaws seen in composite aerospace structures.

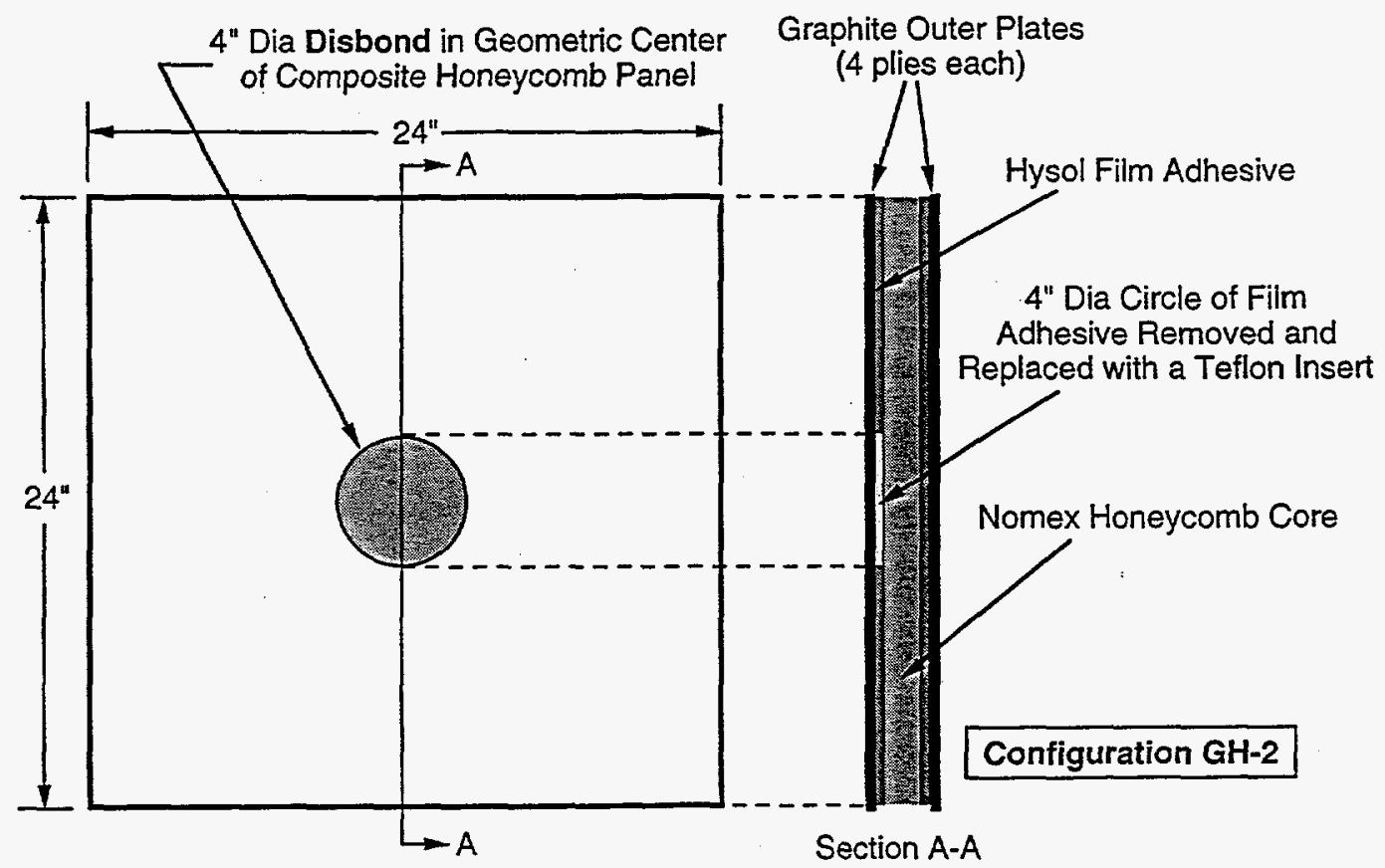

Figure 1. Plate \#2 with Four Inch Diameter Disbond in Center

\section{Diagnostic Measurements}

Diagnostic Measurements which can monitor large areas (from several to hundreds of square feet) on realistic structures (such as aircraft fuselages) with a large number of measurement points (up to 2000) over a large frequency band (up to $2000 \mathrm{~Hz}$ ) are required for performing structural health monitoring via dynamics. Non-contact techniques such as scanning laser vibrometry and laser holography have been used to perform these functions. Zonic A\&D's LAZON system was used as the data acquisition system for the diagnostic measurements discussed in this work. This system consists of two major hardware components: an Ometron Scanning Laser vibrometer and a Zonic Workstation 7000. The Workstation 7000 is a multichannel, real time, FFT-based analyzer and data acquisition system. The System 7000 uses three analog output channels. Channels one and two are used to drive the horizontal and vertical positions of the laser beam. Channel three provided a random output signal to drive a Wilcoxin hybrid piezoelectric/electro-mechanical shaker. Force was input to the panel via an acrylic stinger, a $5 \mathrm{lb}$ load cell, an aluminum pad, dental cement, and aluminum tape. Three analog input channels were also used on the Workstation 
7000. The first channel acquired information from the load cell. The second channel acquired information from a driving point accelerometer. And the third channel acquired all velocity data from the vibrometer.

A measurement grid of 23 by 23 scan points ( 529 total points) on one inch centers and a .5 inch border was used on the plate. The scanning vibrometer allows an order of magnitude increase in the number of measurement points over traditional discrete sensors with a marked decrease in set-up time. This makes it a unique device for obtaining high density (both spatial and frequency) measurements for structural health monitoring applications. A velocity over force Frequency Response Function (FRF) and the associated coherence function were calculated and saved for each scan point. The data set discussed herein utilized a bandwidth of $0-2500 \mathrm{~Hz}$ which is much broader than is typically acquired by traditional accelerometers. A block size of 1024 with 10 averages and 50\% overlap were used for FRF calculations. The data acquisition for each plate takes about 1.5 hours. Figure 2a provides a photograph of the test setup used for the composite plates as seen from the side. The plate is seen suspended in a free-free fashion and covered with white dye penetrant to enhance the laser reflectivity. The shaker and stinger are also seen. Figure $2 b$ shows the same configuration as seen from the rear. The scanning laser vibrometer is seen facing the plate.

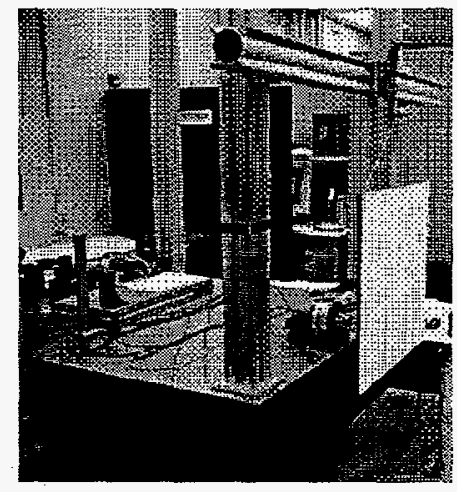

Figure 2a. Experimental Configuration for Composite Plates (Side View)

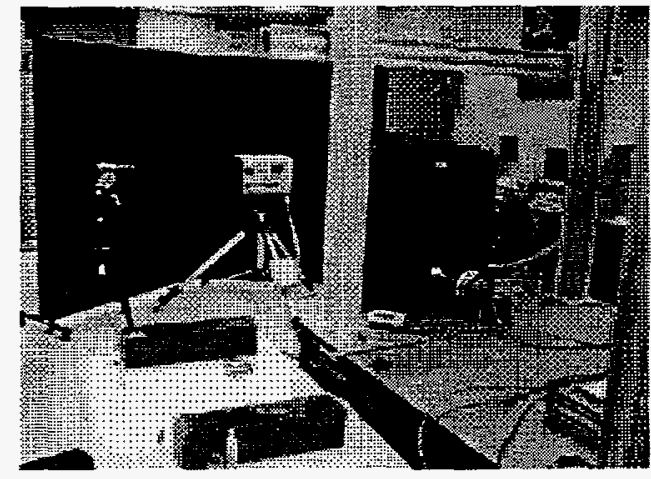

Figure 2b. Experimental Configuration for Composite Plates (Rear View)

\section{Information Condensation}

The amount of data which is generated from 530 measurement points (counting the driving point accelerometer) in the range of 0 to $2500 \mathrm{~Hz}$ with up to 100 significant modes is staggering when conventional processing techniques are applied. Therefore, more automated and robust techniques are needed to process the data and provide the necessary parameters to perform damage identification. Also, techniques which use mode-to-mode comparisons for damage identification are not inherently automatable. Hence, numerical manipulations which combine all the modal information are most useful. One such manipulation entails collecting the modal information into experimental dynamics matrices (mass, damping, and stiffness matrices) [1,2]. These entities combine the modal information into a form which is amenable to more detailed analyses by damage identification algorithms as will be mentioned in the next section. The data is currently being processed into such forms.

Another class of mathematical entities have proven extremely useful for rapid visualization of changes due to damage and have been applied to this data set. Flexibility shapes are a linear combination of all modes in the data and are more robust and sensitive to the damage than individual mode shapes [3]. This idea has been expanded to create a full flexibility matrix [4] which is an inverse of the stiffness matrix. Such an approach provides a robust reduction of the data which maintains the local shape information in a frequency-independent form. Hence mode-to-mode comparisons before and after damage are not required. Also, the system can be "over-identified" meaning that such anomalies as split modes, noise modes, or false modes due to nonlinearities have much less effect on the final data form [5]. Hence, the procedure becomes much more automatable. This processing has been completed for the plate data and is discussed next. 
Figure 3a shows the diagonal values of the flexibility matrix (called driving-point flexibilities) for plate \#1 (undamaged) plotted as a mesh ( $\mathrm{z}$ axis) over the geometric location ( $\mathrm{x}$ and $\mathrm{y}$ axis) on the plate. Physically, these values represent the displacement which would result for a unit force at each scan location. This is an enlightening and rapid method for visualizing the information available in the flexibility matrix. The processing needed to obtain this plot was approximately 20 minutes. In general, the flexibilities of the plate are uniform. Figure $3 \mathrm{~b}$ shows the driving point flexibilities for plate \#2 (disbond). The plate is seen to be much more flexible than plate \#1. However, specific location of the damage is not available with this level of processing. The asymmetric nature of the response is due to boundary condition effects, specifically the stiffening which results from the stinger attachment. It should be noted that these plots represent only the diagonal values of the flexibility matrix. Much more information is available on the off-diagonal terms which relate displacement at each scan point to a unit input force at another scan point. Part of the on-going work is to interpret the entire flexibility matrix and will be mentioned in the next section.

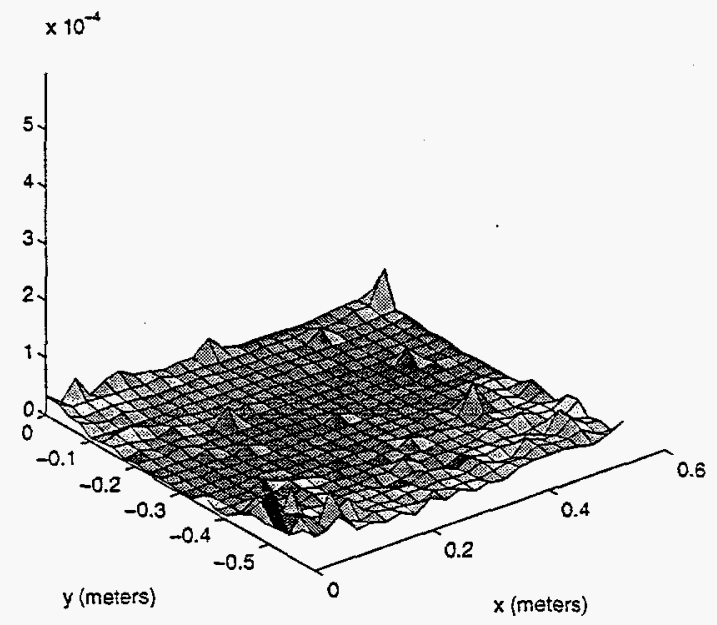

Figure 3a. Driving-Point Flexibilities for
Plate \#1 (Undamaged)

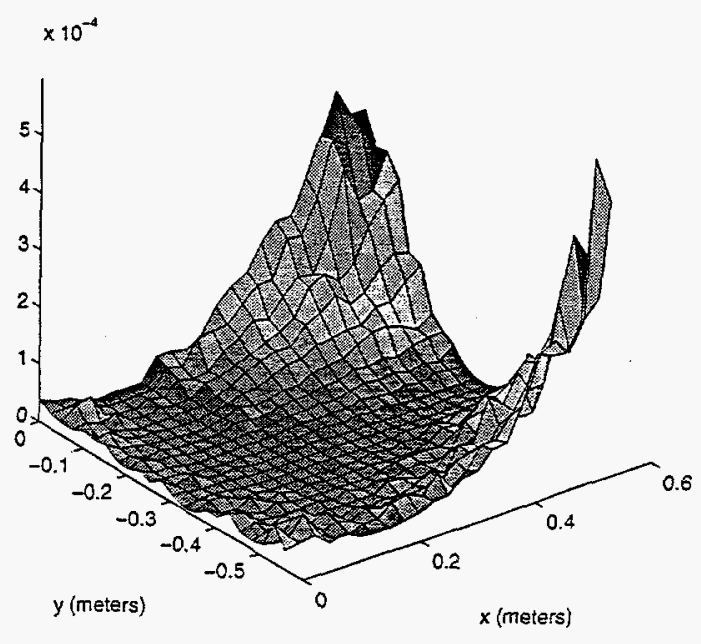

Figure 3b. Driving-Point Flexibilites for Plate \#2 (Disbond in Center)

The driving-point flexibilites for the plate \#3 (fluid-filled section) show a change in the flexibility when compared to plate \#1. Since the mass properties changed, the mode shapes (and the reconstructed flexibility matrix) which changed as well. The driving-point flexibilities for plate \#4 (delamination) shows the least change from plate \#1. This suggests that the delamination flaw provides less of an impact on the stiffness properties of the plate than the disbond (at least in the frequency band measured). It could be expected that this flaw would provide a greater effect on some of the higher modes. In fact, it is presumed that the detached section of the outer plies should have a local resonance. The driving-point flexibilities for plate \#5 (all damage cases) show flexibility changes that are not as great as with plate \#2. However, the center of this plate is intact as opposed to plate \#2. Hence, location of the flaw has an impact on the results at this level of processing. Both plate \#3 and plate \#5 contain a fluid filled section and both show an increase in the magnitude of the flexibility values at the corner nearest the driving point. This is opposite of what is seen in the other three plates. This suggests that mass property changes may contain a unique signature. Again, it must be noted that this is only the initial cursory survey of the data. More advanced processing is required to determine specific information about the different flaw scenarios. This processing is underway and will be discussed in the next section.

\section{Damage Identification}

Damage Identification is the process of operating on the experimental data reduced using techniques described in the previous section to detect, localize, and calculate the extent of the damage. Current work is underway to disassemble the stiffness (or flexibility) matrix to determine localized stiffness parameters. The magnitudes of these parameters will then be compared before and after damage [6]. This takes into account all off-diagonal terms which were ignored in the previous driving-point flexibility analysis. Other damage detection techniques which are under consideration 
include the Minimum Rank Perturbation Theory (MRPT) [7], Structural Translation and Rotation Error CHecking (STRECH) [3], or Strain Energy Comparisons [8].

\section{Conclusions}

The development of a structural health monitoring capability using dynamics involves four tasks: operational evaluation, diagnostic measurement, information condensation, and damage identification. This process has begun for composite aerospace structures. The initial work in operational evaluation has centered around the creation of five composite plates with engineered flaws. Diagnostic measurements using a scanning laser vibrometer have been performed using 529 scan points and a frequency range of 0 to $2000 \mathrm{~Hz}$. This information has been condensed into experimental flexibility matrices. An initial study of the flexibility shapes reveals detectable changes in the plates for disbonds and fluid retention. Continuation of this work will use damage identification techniques to obtain more quantitative information on the existence, location and extent of damage. Follow-on work will use accelerated tests such as resonant fatigue testing to study damage accumulation followed by development of field testing procedures.

\section{Acknowledgments}

Two individuals are providing much insight and direction to this work: Professor Roberto Osegueda of the Civil Engineering Department at the University of Texas at El Paso and Professor Lee Peterson of the Aerospace Sciences Department at the University of Colorado at Boulder. These individuals are thesis advisors for the fourth and fifth authors and are having a significant impact on this work. This work has been supported by the United States Department of Energy under contract DE-AC04-94AL85000.

\section{References}

1. K.F. Alvin, "Second-Order Structural Identification via State Space-Based System Realizations", CU-CSSC-93-09, Ph.D. Dissertation, Aerospace Engineering Sciences, University of Colorado at Boulder, Boulder, CO, April, 1993.

2. G.H. James, T.G. Carne, B.D. Hansche, R.L. Mayes, G.M. Reese, and T.W. Simmermacher, "Health Monitoring of Operational Structures - Initial Results", Proceedings of the 1995 Adaptive Structures Conference, New Orleans, LA, April 13-14, 1995.

3. R.L. Mayes, "An Experimental Algorithm for Detecting Damage Applied to the I-40 Bridge Over the Rio Grande", Proceedings of the 13th International Modal Analysis Conference, Nashville, TN, January 31, 1995.

4. S.W. Doebling, "Measurement of Structural Flexibility Matrices for Experiments with Incomplete Reciprocity", CU-CAS-95-10, Ph.D. Dissertation, Aerospace Engineering Sciences, University of Colorado at Boulder, Boulder, CO, April, 1995.

5. N.A. Robinson, L.D. Peterson, G.H. James, and S.W. Doebling, "Damage Detection in Aircraft Structures Using Static Flexibility Matrices", Proceedings of the 14th International Modal Analysis Conference, Dearborn, MI, February 12-14, 1996.

6. L.D. Peterson, K.F. Alvin, and S.W. Doebling, "Experimental Determination of Local Structural Stiffness by Disassembly of Measured Stiffness Matrices", Proceedings of the 36th SDM Conference, New Orleans, LA, April, 1995.

7. M. Kaouk and D.C. Zimmerman, "Structural Damage Assessment Using a Generalized Minimum Rank Perturbation Theory", Proceedings of the 34th SDM Conference, La Jolla, CA, April, 1993.

8. C.J. Carrasco, "Damage Detection in Beams using Modal Energy Estimates", M.S. Thesis, Civil Engineering Department, The University of Texas at El Paso, El Paso, TX, May, 1992. 


\section{DISCLAIMER}

This report was prepared as an account of work sponsored by an agency of the United States Government. Neither the United States Government nor any agency thereof, nor any of their employees, makes any warranty, express or implied, or assumes any legal liability or responsibility for the accuracy, completeness, or usefulness of any information, apparatus, product, or process disclosed, or represents that its use would not infringe privately owned rights. Reference herein to any specific commercial product, process, or service by trade name, trademark, manufacturer, or otherwise does not necessarily constitute or imply its endorsement, recommendation, or favoring by the United States Government or any agency thereof. The views and opinions of authors expressed herein do not necessarily state or reflect those of the United States Government or any agency thereof. 